\title{
A Physician's Perspective on Generic Drug Quality
}

\author{
Harry M. Lever, MD, FACC, FACP
}

Lever's Viewpoint

$\mathrm{M}$

y perspective on the quality of internationally sourced generic drugs is that of a practicing cardiologist at a tertiary care center that treats patients from around the world, many of whom are quite fragile and dependent on a combination of medication and surgery. I have experienced repeated instances of inconsistent results from how my patients respond to

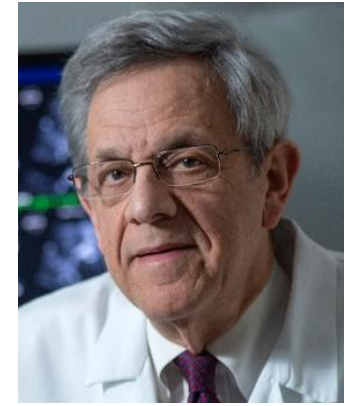

that has subsequently been expanded 51 times to include at least 10 companies. It is estimated that drugs sold to millions of patients in 30 countries may have been compromised.

I recently conducted an internet search of recalled angiotensin receptor blockers on the FDA's website and found 1,159 recalled lots as of July 2019. This has made it hard to write prescriptions for this class of drugs because at any one time it is difficult to know which lots have been contaminated. generic medications, particularly those originating in poorly regulated countries such as China and India. My experiences were documented in Katherine Eban's explosive expose on the international generic drug industry, Bottle of Lies. ${ }^{1}$

I have had patients stabilized on diuretics in the hospital then discharged, only to be quickly readmitted with heart failure. I have also had ambulatory patients who have been stable for a prolonged period on a diuretic suddenly go into heart failure. When I investigated these problems, I have routinely found that my patients had recently been switched to a new generic source of their diuretic. By simply changing the medication's manufacturer back to what it was before, while keeping the dose the same, I have found these patients immediately improved.

I have also observed problems with antihypertensive drugs in which a patient is started on one manufacturer's version then changed by the pharmacy to another and their blood pressure suddenly spikes. When that occurs, therapy with other drugs may be initiated that subjects the patient to additional cost and risks.

Recently, there has been a problem with contamination of at least 3 angiotensin receptor blockers with N-Nitrosodimethylamine (NDMA), a probable human carcinogen. ${ }^{2}$ This apparently resulted from a manufacturing change by the drug maker Zhejiang Huahai, one of China's biggest generic drug manufacturers. Apparently, the company decided there was too much waste in the manufacturing of these drugs and chose to use a NDMA-containing solvent that was once an additive to rocket fuel to accelerate extraction of the active ingredient. Unfortunately, the chemical was not removed when the process was complete. The change in the formulation was made in 2011 but was not detected until July 2018, when the U.S. Food and Drug Administration (FDA) initiated a recall

J Manag Care Spec Pharm. 2020;26(5):592-93

Copyright $\odot 2020$, Academy of Managed Care Pharmacy. All rights reserved.
The testing of individual lots of internationally sourced generic drugs for potency and purity is not consistently done, and even when it is, it is not reported to practicing physicians. It is also difficult for the physician or patient to determine which manufacturer's lot the patient might have received because pharmacies are not required to put the lot number on the individual prescription container. Since the manufacturing problems relative to NDMA contamination are now apparent, one would expect that the problem would have been corrected and there would be no more recalls of these drugs. However, the problem continues for reasons that are not clear. As a result, I have found myself writing multiple prescriptions for the same drug because of the multiple recalls.

Another problematic drug in my practice is the beta blocker metoprolol succinate, which is a sustained release drug for the treatment of patients with hypertrophic cardiomyopathy, as well as angina pectoris and hypertension. This drug is particularly hard to reproduce because it is a sustained release formulation and the proprietary information about the original formulation is not available to generic manufacturers. For many patients, I have found that only the authorized generic or the name brand drug work consistently.

As the physician responsible for the care of my patients, the question I am too often left with is this: Am I dealing with a patient whose disease is simply not responding to medical treatment or with a poor quality drug? In addition to putting patients unnecessarily at risk, this increases the cost of care, which would appear to be the antithesis of what managed care is supposedly all about. Case in point: hospitals in the United States pay fines to the Centers for Medicare \& Medicaid Services when Medicare patients are readmitted within 30 days of discharge for heart failure.

When I recognized that there was a clinical problem with metoprolol succinate made by the Indian manufacturer Wockhardt, I reported it to the FDA. I was told they would investigate my concerns and get back to me. It took them 
18 months to do so, and when they finally did, I was told there was no problem. After reading Bottle of Lies, however, I learned that an FDA inspector was in the plant 7 months after I wrote my letter and had found severe problems. About 3 months after I received the FDA letter, it was then reported in the press that indeed there was a problem with this generic version and it was removed from the market because of dissolution problems.

A particularly severe problem involves the use of the drug tacrolimus, which prevents organ rejection after heart transplant. Our team at the Cleveland Clinic has observed a number of patients who suddenly began rejecting their new hearts after the manufacturer of the drug had been changed. Clearly, with such complicated patients this should simply never happen.

From my perspective, it appears that when it comes to generic drugs, the primary consideration of managed care is cost. While I understand that brand-name drugs have become very expensive, there has to be a rational middle ground. With the importation of drugs from poorly regulated countries such as India and China, the FDA is simply not doing its job. Although it is commonly believed that the FDA does a lot of testing of drugs, in reality they are usually just accepting data from drug companies, some of which routinely falsify their data, according to Ms. Eban's book.

Because the conditions under which internationally sourced drugs are transported can vary dramatically, these conditions should be rigorously regulated and that regulation strictly enforced. Furthermore, quality and potency testing should be required for each lot of imported generic drugs. This is clearly possible as has been demonstrated by Valisure Pharmacy in New Haven, CT, which tests every lot of drugs they sell without imposing a significant increase in the price.

In summary, the following are my recommendations for how clinicians, patients, and health care managers can mitigate the threat of inconsistent generic drug quality:

- Recognize that not all generics marketed in the United States are of the same quality and that the FDA is either unwilling or unable to adequately ensure that they are.

- When treating a patient who suddenly changes clinical status, consider checking to determine if there has been a recent change in the manufactured source of the patient's medications.

- Make patients aware of possible generic drug quality issues and encourage them to inspect their pills before leaving the pharmacy. If they are different, they should ask for an explanation from the pharmacist.

- Patients should insist that their pharmacies alert them whenever the manufactured source of a generic drug they are taking changes. This information should also be relayed to the prescriber, either by the patient or the pharmacy. Ideally, the pharmacy would be required to alert the prescriber, as is the case in Utah when a pharmacy dispenses a different manufacturer's antiseizure medication.

- Patients and prescribers should be taught to use internet- based medication identifiers such as Drugs.com to confirm the identity of their medication, and DailyMed.com to determine the different available generic sources of the medication in the United States. If all else fails, call the company listed on the medication's original packaging and ask where the finished dosage form and all active ingredients are made. You will be surprised to learn that the "manufacturer" listed on the original container is often just a U.S. distributor for a foreign manufacturer, often in India. A quick check with the FDA (https://www.fda.gov/safety/recalls-market-withdrawals-safety-alerts) will determine whether the company in question has been the subject of a recall, market withdrawal, or safety alert.

- Try to stay up to date on quality-related pharmaceutical issues.

- Consider creating your own list of reliable generic drug manufacturers and/or those that have demonstrated a history of quality problems, as we have done at the Cleveland Clinic, as described in Ms. Eban's book.

Finally, it seems clear to me that the ingredient sourcing and manufacturing of generic prescription drugs should be brought back to this country to provide more security and control over the finished products we provide to patients. We must reduce our dependency on foreign ingredient sources and manufacturers. This will require that the medical profession, the drug industry, the insurance companies, and the government work together as partners. While not easy, I believe it is necessary to ensure the safety and effectiveness of this vital part of contemporary medicine.

\section{Authors}

HARRY M. LEVER, MD, FACC, FACP, is Medical Director for the Hypertrophic Cardiomyopathy Clinic at the Cleveland Clinic, Cleveland, Ohio.

AUTHOR CORRESPONDENCE: Harry M. Lever, MD, can be contacted at 216.444.6970 or leverh@ccf.org.

\section{DISCLOSURES}

No funding supported the writing of this article. The author has nothing to disclose.

\section{REFERENCES}

1. Eban K. Bottle of Lies: The Inside Story of the Generic Drug Boom. New York: HarperCollins Publishers; 2019.

2. Edney A, Berfield S, Yu E. Carcinogens have infiltrated the generic drug supply in the U.S. Bloomberg Business Week. September 12, 2019. Available at: https://www.bloomberg.com/news/features/2019-09-12/how-carcinogentainted-generic-drug-valsartan-got-past-the-fda. Accessed March 30, 2020. 